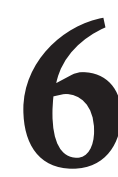

\title{
ANÁLISIS DEL TRABAJO FIN DE GRADO EN MAGISTERIO, EDUCACIÓN SOCIAL Y PEDAGOGÍA EN ESPAÑA
}

\author{
(ANALYSIS OF THE DEGREE FINAL PROJECT IN EDUCATION, SOCIAL \\ PEDAGOGY AND PEDAGOGY IN SPAIN)
}

María José Chisvert-Tarazona

Raúl Tárraga-Mínguez

Alicia Ros-Garrido

Davinia Palomares-Montero

Universitat de València

DOI: 10.5944/educXX1.28572

\section{Cómo referenciar este artículo/How to reference this article:}

Chisvert-Tarazona, M., Tárraga-Mínguez, R., Ros-Garrido, A., y Palomares-Montero, D., (2021). Análisis del Trabajo de Fin de Grado en Magisterio, Educación Social y Pedagogía en España. Educación XX1, 24(2), 143-166. https://doi.org/10.5944/educXX1.28572

Chisvert-Tarazona, M., Tárraga-Mínguez, R., Ros-Garrido, A., \& Palomares-Montero, D., (2021). Analysis ot the Degree Final Project in Teacher Education, Social Pedagogy and Pedagogy in Spain. Educación XX1, 24(2), 143-166. https://doi.org/10.5944/educXX1.28572

\section{RESUMEN}

El Trabajo Fin de Grado (TFG) se considera la experiencia de aprendizaje más relevante de un programa académico, de hecho, incluso se utiliza como indicador de la calidad del grado en su conjunto. Dada su relevancia, el objetivo de este estudio es analizar el planteamiento que realizan las universidades públicas y privadas en España de la asignatura de TFG de los grados en Maestro/a en Educación Infantil y/o Primaria, en Educación Social y en Pedagogía. Para alcanzar este objetivo se ha llevado a cabo un análisis de contenido de las 173 guías docentes de la asignatura de TFG en los grados del ámbito de la educación de las universidades españolas; y se ha realizado un grupo de discusión en el que han participado siete expertos en gestión y/o investigación en TFG. Los resultados ponen en evidencia diferencias significativas entre universidades públicas y privadas en aspectos 
como la carga de créditos de la asignatura, los elementos evaluables del trabajo, así como los agentes que lo evalúan. Los expertos participantes en el grupo de discusión consideran que el TFG debe ser un instrumento que ponga en evidencia si las competencias del grado han sido adquiridas. El análisis global de los resultados sugiere la necesidad de planificar el currículum de la asignatura de TFG de forma flexible, adaptar la carga de créditos a las exigencias del trabajo a realizar, así como diversificar la tipología de TFG para que los estudiantes escojan cuál realizar. Se concluye el interés de acordar las directrices generales de la labor tutorial, adoptar un modelo de evaluación formativa que proporcione un feedback valioso para el alumnado y tomar como referente del TFG el perfil profesional, para promover un aprendizaje facilitador del desempeño en el contexto laboral.

\section{PALABRAS CLAVE}

Análisis de contenido, educación superior, grupo de discusión, guías docentes, planificación curricular, Trabajo Fin de Grado

\section{ABSTRACT}

The Degree Final Project (DFP) is considered the most relevant learning experience of an academic program. In fact, it is even used as an indicator of the degree's quality. Given its relevance, this study aims to analyse Spanish public and private university approaches to the DFP for Degree in Early Childhood Education Teachers and / or Primary Education Teachers, Degree in Social Education and Degree in Pedagogy. We have conducted a content analysis of the 173 DFP's teaching guides in the degrees of education at Spanish universities. Moreover, a focus group has been held in which seven experts in management and / or research in DFP have participated. Findings show significant differences between public and private universities in some aspects such as the academic credit load, the assessable items, as well as the agents that evaluate the DFP. Experts in the focus group consider that the DFP should be a tool that shows whether knowledge and competences have been acquired during the degree course. Results suggest the need to plan the DFP's curriculum in a flexible way, adapting the academic credit load to the demands of the task required, as well as diversifying the type of DFP so that students choose which one to do. We conclude that it is necessary to agree on the general guidelines of the tutorial work, to take a formative assessment model that provides valuable feedback to the students and to take the professional profile as a reference for the DFP in order to foster facilitative learning of performance in the work context. 
MARÍA JOSÉ CHISVERT-TARAZONA, RAÚL TÁRRAGA-MÍNGUEZ, ALICIA ROS-GARRIDO

\section{KEY WORDS}

Content analysis, higher education, discussion groups, curriculum guides, educational planning, degree final project

\section{INTRODUCCIÓN}

Los nuevos modos de comunicación y acceso al conocimiento, la exponencial generación de saber en una sociedad altamente tecnologizada e interconectada, así como la precarización del trabajo, en tiempos de incertidumbre en los que las decisiones se ramifican y no son definitivas (Bauman y Leonci, 2018), han impulsado el requerimiento de una revisión del currículum en los sistemas universitarios (Rekalde, 2011).

El Espacio Europeo de Educación Superior (EEES) propicia una nueva ordenación de enseñanzas universitarias dirigidas a flexibilizar su organización y a posibilitar la diversificación curricular. Sus directrices han obligado a repensar los modelos pedagógicos universitarios, más centrados, en la actualidad, en los resultados de aprendizaje y en generar las condiciones para que se alcancen. Sin embargo, a medida que los métodos de enseñanza y aprendizaje se vuelven más heterogéneos, la medición y comparación de las competencias generales obtenidas en los grados universitarios resulta más difícil (Fretwell, 2003). Los nuevos sistemas de calidad introducidos en las universidades europeas se encuentran en la tesitura de demostrar que el estudiantado graduado ha adquirido las competencias establecidas en su área de conocimiento. En este contexto, la aparición de asignaturas como el Trabajo Fin de Grado (TFG) plantea nuevos retos curriculares para la mayoría de los equipos de gobierno y personal académico, que cada vez deben atender en mayor medida a demandas globales (Rosenmund, 2006).

En este contexto, el TFG se considera la experiencia de aprendizaje más relevante del programa académico; incluso se utiliza como indicador de la calidad del grado en su conjunto (Jawitz et al., 2002).

En ocasiones resulta difícil, incluso para el propio profesorado, diferenciar estos trabajos de culminación de una titulación universitaria de los trabajos fin de máster o de las tesis doctorales (Díaz-Vázquez et al., 2018). Objetivos y competencias deben ser un referente diferenciador. Complementariamente, en el TFG se agudiza la limitación de recursos investigadores del estudiantado; la escasa disposición de tiempo para su ejecución (Anderson et al., 2008); así como las limitadas interacciones con el profesorado que les tutoriza y asesora (Healey et al., 2013).

El TFG es una práctica específica dirigida a que el alumnado universitario de último curso produzca conocimiento y relacione e integre los saberes adquiridos a lo largo de la titulación desde la autonomía. Nace con voluntad 
de poner a prueba en el estudiantado la capacidad de investigar problemas, de emitir juicios sobre la base de pruebas sólidas, de tomar decisiones de manera racional y de comprender lo que está haciendo. Como sostiene Brew (2007), este trabajo beneficia un proyecto vital y profesional, no solo una carrera académica.

Los TFG se han visto con frecuencia como una oportunidad para que el estudiantado adquiera sus primeras habilidades de investigación (Cuthbert, 1995). Para el alumnado con mejores expedientes académicos, puede convertirse también en una posibilidad para desarrollar trabajos de posgrado (Mateo et al., 2012), si bien la visión actual de estos proyectos es más amplia. Se considera como una experiencia de aprendizaje importante en sí misma: aproxima a la investigación, ofrece una oportunidad para que el alumnado aborde áreas problemáticas en el ámbito profesional y/o permite el desarrollo de proyectos de revisión de literatura académica.

Generalmente, la elaboración del TFG es un proceso individual cuya principal intención es la de aglutinar el acervo competencial propio del grado, una aspiración que necesariamente está condenada a convivir con las limitaciones prácticas que imponen las circunstancias en que debe realizarse. Circunstancias como la de ser un trabajo de una amplitud inusual si se compara con otras prácticas requeridas durante el grado, que se desarrolla en plazos de tiempo escasos y que requiere de aprendizajes y recursos no siempre disponibles por parte del alumnado. Dificultades que limitan las oportunidades del estudiantado de vincularse a un auténtico proceso de generación de conocimiento (DíazVázquez et al., 2018).

En el contexto español, el artículo 12.7 del Real Decreto 1393/2007, de 29 de octubre, introduce algunos determinantes curriculares: "tendrá entre 6 y 30 créditos, deberá realizarse en la fase final del plan de estudios y estar orientado a la evaluación de competencias asociadas al título" (Boletín Oficial del Estado, 2007, p. 44040). Complementariamente, y con la finalidad de unificar criterios y procedimientos dirigidos a una planificación y evaluación del TFG homogénea, muchas universidades optan por el desarrollo de normativa propia. Se trata de los primeros niveles de concreción curricular.

Investigaciones previas en el contexto español constatan la preocupación relativa al qué, profundizando en el análisis competencial y de resultados de aprendizaje (Manzanares-Moya y Sánchez-Santamaría, 2016; Molina et al., 2020; Tejada y Ruiz, 2016); al cómo, desde el análisis de la tutorización y asesoramiento (Díez, 2015; Manzanares-Moya y Sánchez-Santamaría, 2016; Vera y Briones, 2015); y a los procesos de evaluación (Mateo et al., 2012; SánchezSantamaría, 2017; Vera y Briones, 2015). También podemos encontrar guías para facilitar su elaboración, como la propuesta por Sánchez et al. (2016), así como investigaciones que se centran en la visión del estudiante sobre este trabajo (Rodríguez et al., 2019). 
En este contexto, el objetivo de este estudio es analizar el planteamiento que realizan las universidades públicas y privadas en España de la asignatura de TFG de los grados en Maestro/a en Educación Infantil y/o Primaria, en Educación Social y en Pedagogía. Objetivo necesario actualmente, ya que plantea un análisis que hasta el momento ha sido escasamente abordado desde el ámbito de investigación de nuestro contexto, pese a que se trata de una asignatura que ya acumula un amplio recorrido en nuestro sistema universitario.

\section{METODOLOGÍA}

En el presente estudio se ha llevado a cabo un análisis multimetodológico, en dos fases, para tratar de dotar de validez los resultados obtenidos. En la primera fase se aplica una metodología evaluativa para conocer cómo se planifica el currículum del TFG a través de las guías docentes de los grados de Maestro/a en Educación Infantil y Primaria, Educación Social y Pedagogía. Por tanto, la investigación desarrollada responde a una intencionalidad descriptiva, pues no pretende construir teoría generalizable a otros contextos, sino describir un fenómeno particular como es el diseño y la planificación del TFG en el área de conocimiento de educación. Además, en la segunda fase se lleva a cabo un grupo de discusión con responsables académicos vinculados a la coordinación o estudio del TFG en el contexto español. Así, los resultados obtenidos en la fase previa podrán ser revisados a tenor de la experiencia práctica de personas directamente vinculadas a la planificación y/o estudio del TFG.

El planteamiento del estudio en dos fases determina la necesidad de utilizar estrategias específicas de recogida y análisis de la información.

\section{Análisis de contenido}

El análisis de contenido (Krippendorff, 1990) es de tipo cualitativo, permite obtener un conocimiento en profundidad de los valores presentes en mensajes, textos o discursos (Gervilla-Castillo, 2004), tanto explícitos como latentes (Carlós y Telmo, 2000) y analiza las condiciones mismas en que se han producido. Esta modalidad mixta, tiene en cuenta tanto la frecuencia de aparición de los indicadores como la presencia o ausencia de los mismos.

Tras realizar una búsqueda de la oferta de estudios de las 50 universidades públicas y 26 privadas de nuestro sistema educativo (CRUE, 2020), se identificaron un total de 173 grados de las 4 titulaciones revisadas, cuya distribución por grado universitario y titularidad de la universidad se reflejan en la Tabla 1. Debe considerarse que aunque los grados de Maestro/a en Educación Infantil y en Educación Primaria son dos titulaciones diferentes, su análisis se ha abordado de manera conjunta. En la primera fila de la Tabla 
1 se hace referencia a ambos debido a que en la mayoría de los casos (57 de 60 universidades) una misma institución oferta los dos grados simultáneamente, con una evidente similitud entre las dos guías. Al compararlas en cada universidad, se constata que el contenido es prácticamente idéntico en todos los casos en referencia a los agentes evaluadores y los elementos evaluados. Únicamente se hallaron diferencias en el tipo de competencias propuestas en siete universidades (11.66\%); y en el número de créditos otorgados a los dos TFGs en una universidad.

Tabla 1

Universo de universidades en España en las que se cursan grados de Educación

\begin{tabular}{llll}
\hline \multicolumn{1}{c}{ Grado } & Univ. públicas & Univ. Privadas & \multicolumn{1}{c}{ Total } \\
\hline Maestro/a en E. Infantil y E. Primaria & $39(65.0 \%)$ & $21(35.0 \%)$ & 60 \\
\hline Educación Social & $28(82.4 \%)$ & $6(17.6 \%)$ & 34 \\
\hline Pedagogía & $18(81.8 \%)$ & $4(18.2 \%)$ & 22 \\
\hline Total & $85(73.7 \%)$ & $31(26.3 \%)$ & 116 \\
\hline
\end{tabular}

Fuente: Elaboración propia

Una vez identificados los grados universitarios, se accedió, a través de la página web, a las 173 guías docentes de la asignatura de TFG para su revisión (no se realizó el análisis de las guías docentes de TFG de dobles grados o grados bilingües).

Se identificaron distintos niveles de análisis que abarcan tres tipos de datos (Palomares-Montero y Chisvert-Tarazona, 2014): unidades de muestreo (registros documentales que conforman la realidad a investigar, es decir, las guías docentes de la asignatura de TFG en los grados analizados), unidades de contexto (texto que identifica la información que se utiliza para describir las unidades de registro, es decir, secciones recurrentes que aparecen en las guías docentes: características generales, tipología, formato, metodología, competencias, relación con prácticum y evaluación de los TFG) y unidades de registro (contenido que puede ser codificado, cuya selección en el presente estudio se basó en la concreción de palabras clave atendiendo otras propuestas de la literatura como Díaz-Vázquez et al. (2018), Fraile et al. (2018), ManzanaresMoya y Sánchez-Santamaría (2016) y Sánchez et al. (2016). La Tabla 2 muestra las unidades de registro aplicadas en el estudio tras su validación en estudio piloto previo por parte del equipo de investigación. 
Tabla 2

Unidades de registro utilizadas en el análisis de contenido de las guías docentes de TFG

\begin{tabular}{ll}
\hline \multicolumn{1}{c}{ Unidades de contexto } & \multicolumn{1}{c}{ Unidades de Registro } \\
\hline Características generales & $\begin{array}{l}\text { Carga lectiva, créditos, temporalización (cuatrimestral, } \\
\text { anual), prerrequisitos (matrícula, depósito, formación } \\
\text { previa). }\end{array}$ \\
\hline Tipología y formato & $\begin{array}{l}\text { Investigación, intervención, revisión teórica, proyecto } \\
\text { técnico, temáticas, individual, grupal. }\end{array}$ \\
\hline Metodología: acción tutorial & $\begin{array}{l}\text { Tutorización, asignación docente, carga docente, } \\
\text { formación previa, continuidad/estabilidad }\end{array}$ \\
\hline Competencias & Generales, específicas, transversales, instrumentales \\
\hline Relación con el prácticum & Prácticum, prácticas, perfil profesional \\
\hline Evaluación & $\begin{array}{l}\text { Agentes: tutor/a, tribunal, autoevaluación } \\
\text { Elementos: informe (inicial, intermedio, final), defensa, } \\
\text { rúbrica, calificación }\end{array}$ \\
\hline
\end{tabular}

Fuente: Elaboración propia

Tras la realización del análisis de contenido, se calculó el estadístico chi cuadrado en las variables cuyo análisis se consideró relevante desde el punto de vista teórico, con el objetivo de determinar si su frecuencia era homogénea (o por el contrario se daba una distribución diferente), tanto en las cuatro titulaciones revisadas, como en función de la titularidad pública o privada de las universidades.

\section{Grupo de discusión}

Tras el análisis de las guías docentes, se generó un espacio de discusión académica con personas responsables de la coordinación y/o estudio del TFG en torno a los resultados obtenidos. Este análisis permitió supervisar la validez de contenido de los resultados, para lo cual se remitió a los participantes del grupo de discusión, previo a la realización del encuentro virtual, un breve informe de resultados mostrando la frecuencia de las unidades de registro en las distintas unidades de contexto (recogidas en Tabla 2).

La selección de las personas participantes se realizó mediante una captación activa que respondía a un muestreo intencional (Perelló, 2009), para asegurar la representatividad de personas expertas en gestión y/o investigación en TFG de educación, con vinculación a universidades públicas y privadas, así como a los cuatro grados objeto de análisis. En julio de 2020, se contaba con el compromiso manifiesto de diez personas que respondían a este criterio de inclusión. Sin embargo, el desarrollo del grupo de discusión, realizado virtualmente en septiembre de 2020, contó con la participación final de siete 
participantes. Su distribución por grado es la siguiente: tres participantes tienen responsabilidades en el TFG del grado de Educación Social (GES), tres en el TFG del grado de Pedagogía (GP) y una en el TFG de los grados de Maestro/a (GM). En todos los casos, las personas participantes cuentan con experiencia docente en los grados objeto de estudio. En particular, seis en Educación Social, cinco en los grados de Magisterio y cuatro en Pedagogía. Adicionalmente, tres de ellas son personas que han elaborado reconocidos trabajos de investigación relacionados con la planificación y/o implementación del TFG.

El grupo de discusión, de una hora y 40 minutos de duración, fue conducido para facilitar el debate discursivo. Para ello, se hizo uso de un guion flexible de preguntas que revisaban los resultados obtenidos en la fase previa de la investigación atendiendo las distintas unidades de contexto (recogidas en Anexo 1).

Su grabación posibilitó la transcripción en formato texto de manera naturalista, respetando la jerga propia. En el proceso de análisis se establecieron criterios temáticos agrupados de acuerdo a los contenidos de las preguntas planteadas al grupo, siguiendo las distintas unidades de contexto y replicando la codificación de las unidades de registro realizada en la fase previa mostrada en la Tabla 2. El análisis de contenido de los datos, basado en técnicas cualitativas, lo realizaron cuatro investigadores al mismo tiempo, previa lectura individual, para, finalmente, extraer los resultados en base a dos criterios: saturación de las respuestas y relevancia/interés de las mismas.

El estudio presenta, por tanto, una triangulación metodológica a nivel de instrumento (ficha de análisis de contenido y guion de grupo de discusión) y de fuentes de información (guías docentes y personas expertas).

\section{RESULTADOS}

\section{Características generales}

La totalidad de los grados revisados otorgan a la asignatura de TFG una carga lectiva de entre 6 y 14 créditos. La moda se sitúa en los 6 créditos en las cuatro titulaciones.

En las universidades públicas la opción mayoritaria es que el TFG tenga una carga de 6 créditos en todos los grados (Tabla 3), pero especialmente en los grados de Maestro/a (76.9\%) y en Educación Social (75\%).

En el caso de las universidades privadas, en la mitad de las titulaciones analizadas el TFG tiene una carga de 6 créditos y la otra mitad otorga una carga superior a 6 créditos. 
El estadístico chi cuadrado indica que esta diferente tendencia entre las universidades públicas y privadas en la carga lectiva que se otorga al TFG es estadísticamente significativa $\chi^{2}=5.51_{(1)}, \mathrm{p}=.019$. Es decir, las universidades privadas tienden a dar al TFG un número de créditos mayor que las universidades públicas.

Si comparamos el número de créditos del TFG entre las titulaciones (en este caso sin diferenciar entre universidades públicas y privadas), observamos que no hay diferencias estadísticamente significativas $\chi^{2}=1.03_{(1)}, \mathrm{p}=.598$

Tabla 3

Número de créditos del TFG en los grados del área educativa de las universidades españolas

\begin{tabular}{|c|c|c|c|c|c|c|c|}
\hline \multirow[b]{2}{*}{ Créditos } & \multicolumn{2}{|c|}{ Maestro/a } & \multicolumn{2}{|c|}{$\begin{array}{l}\text { Educación } \\
\text { Social }\end{array}$} & \multicolumn{2}{|c|}{ Pedagogía } & \multirow{2}{*}{$\begin{array}{c}\text { Chi } \\
\text { cuadrado }\end{array}$} \\
\hline & 6 & $>6$ & 6 & $>6$ & 6 & $>6$ & \\
\hline $\begin{array}{l}\text { Universidades } \\
\text { públicas }\end{array}$ & $\begin{array}{c}30 \\
(76.9 \%)\end{array}$ & $\begin{array}{c}9 \\
(23.1 \%)\end{array}$ & $\begin{array}{c}21 \\
(75 \%)\end{array}$ & $\begin{array}{c}7 \\
(25 \%)\end{array}$ & $\begin{array}{c}11 \\
(61.1 \%)\end{array}$ & $\begin{array}{c}7 \\
(38.9 \%)\end{array}$ & \multirow{2}{*}{$\begin{array}{c}\text { diferencias } \\
\text { entre } \\
\text { titulaciones } \\
\chi^{2}=1.03 \\
p=.598\end{array}$} \\
\hline $\begin{array}{l}\text { Universidades } \\
\text { privadas }\end{array}$ & $\begin{array}{c}13 \\
(54.2 \%)\end{array}$ & $\begin{array}{c}9 \\
(42.9 \%)\end{array}$ & $\begin{array}{c}1 \\
(16.7 \%)\end{array}$ & $\begin{array}{c}5 \\
(83.3 \%)\end{array}$ & $\begin{array}{c}2 \\
(50 \%)\end{array}$ & $\begin{array}{c}2 \\
(50 \%)\end{array}$ & \\
\hline $\begin{array}{l}\mathrm{T} \text { o t a } 1 \\
\text { (públicas }+ \\
\text { privadas) }\end{array}$ & $\begin{array}{c}43 \\
(70.5 \%)\end{array}$ & $\begin{array}{c}18 \\
(29.5 \%)\end{array}$ & $\begin{array}{c}22 \\
(64.7 \%)\end{array}$ & $\begin{array}{c}12 \\
(35.3 \%)\end{array}$ & $\begin{array}{c}13 \\
(59.1 \%)\end{array}$ & $\begin{array}{c}9 \\
(40.9 \%)\end{array}$ & $\begin{array}{c}\text { públicas vs } \\
\text { privadas } \\
\chi^{2}=5.51_{(1)} \\
p=.019\end{array}$ \\
\hline
\end{tabular}

Fuente: Elaboración propia

Los participantes del grupo de discusión cuestionan el escaso número de créditos concedidos a la asignatura cuando afirman que "la carga lectiva de seis créditos que se recoge en la gran mayoría de los grados es poca" (GP1), así como también que se imparte, mayoritariamente, en el segundo cuatrimestre. De hecho, el TFG es considerado un proyecto de envergadura que debería incorporarse como asignatura anual para responder a las competencias del grado. Esta consideración de asignatura anual supondría también ampliar el número de créditos del TFG, otorgándole mayor importancia y promoviendo un aprendizaje personal de mayor calado si se garantiza regularidad, control y seguimiento:

Convienen los doce créditos por dos razones. Primero porque te permiten plantear ... un TFG anual..., poner sistemas de seguimiento y de control compartidos entre todo el profesorado, tutor y estudiantes para poder hacer seguimiento... Segundo porque... los 12 créditos permiten mayor 
empoderamiento al estudiante, hacer un proyecto personal que sintetice su aprendizaje en la titulación (GP2).

La decisión sobre el número de créditos otorgados a la asignatura de TFG no es trivial, responde a un posicionamiento de la propia institución. Aquellas instituciones que contemplan los TFG con una carga de seis créditos parten de un modelo normativo, donde "el TFG no deja de ser algo residual que había que colocarlo porque la normativa lo establecía” (GES1) y, por tanto, podría adolecer de relevancia curricular en el plan de estudios. Por el contrario, programar los TFG con un elevado número de créditos, más habitual en universidades privadas, representa un modelo preocupado por la rentabilidad en la gestión organizativa. De hecho, "mayor peso del TFG en créditos, en el caso de las universidades privadas donde muchas de ellas son virtuales, favorece que tenga un planteamiento mayor, implica menos contrataciones y tiene consecuencias organizativas" (GES1).

Adicionalmente, parece que la decisión de asignar un número u otro de créditos al TFG no depende exclusivamente de una variable. La tipología de TFG, que analizaremos más adelante, también debería ser considerada en la toma de decisión a este respecto pues "igual el tiempo que tiene que invertir el alumnado si hace una investigación es mayor que si realiza una unidad didáctica" (GM1). En cualquier caso, parece que no es tan relevante el número de créditos asignados al TFG sino "que sea un trabajo con sentido y que sirva de cara a la empleabilidad, al desarrollo profesional" (GP3), aspecto vinculado a la identidad profesional, especialmente en Pedagogía y Educación Social.

En cuanto a los prerrequisitos para la matrícula y el depósito del TFG, el criterio más frecuente que se establece como condición para matricularse del TFG hace alusión al número de créditos aprobados, o a la necesidad de haber superado asignaturas, siendo la más habitual el prácticum.

El grupo de discusión aludió a la necesidad de ofrecer algún tipo de formación sobre el proceso y estructura del TFG: "píldoras formativas para el estudiantado" (GM1), "seminarios" (GP1 y GP3), "una guía” (GES3) o "jornadas" (GES2). Si bien, partir de la expectativa de una falta de competencias específicas para su elaboración puede también considerarse un "paternalismo, al dar respuesta a la poca capacidad que tiene el alumno para asumir retos, desafíos y autonomía" (GES1).

\section{Tipología y formato de TFG}

El análisis de las guías docentes pone en evidencia la diversidad de tipologías para la realización del TFG. Mayoritariamente se ofrecen diversas alternativas entre las que el alumnado puede elegir, sin o con alguna condición. La revisión ha mostrado que las tipologías de TFG más frecuentes son las de 
investigación, intervención, revisión teórica y finalmente, la realización de proyectos técnicos (Tabla 4).

Esta distribución de tipologías de TFG es significativamente diferente entre las universidades públicas y privadas, de manera que en las universidades públicas se ofrece un abanico significativamente más amplio de opciones de tipologías de TFG que en las privadas $\left(\chi^{2}=15.50_{(4)}, \mathrm{p}=.004\right)$. Sin embargo, no existen diferencias entre las tres titulaciones en la distribución de la tipología de $\operatorname{TFG}\left(\chi^{2}=4.37_{(8)}, \mathrm{p}=.823\right)$.

Tabla 4

Tipologías de TFG en función del grado universitario y titularidad de la universidad

\begin{tabular}{|c|c|c|c|c|c|}
\hline & $\begin{array}{l}\text { Investigación } \\
\qquad(\mathrm{n}=95)\end{array}$ & $\begin{array}{c}\text { Intervención } \\
(\mathrm{n}=93)\end{array}$ & $\begin{array}{c}\text { Revisión } \\
\text { teórica } \\
(n=78)\end{array}$ & $\begin{array}{l}\text { Proyecto } \\
\text { técnico } \\
(n=67)\end{array}$ & $\begin{array}{c}\text { Chi } \\
\text { cuadrado }\end{array}$ \\
\hline $\begin{array}{l}\text { Maestro/a } \\
(\mathrm{N}=60)\end{array}$ & $45(75 \%)$ & $45(75 \%)$ & $36(60 \%)$ & $\begin{array}{c}32 \\
(53.3 \%)\end{array}$ & \multirow{3}{*}{$\begin{array}{l}\text { diferencias } \\
\text { entre } \\
\text { titulaciones } \\
\chi^{2}=4.37_{(8)} \\
p=.823\end{array}$} \\
\hline $\begin{array}{l}\text { Educación } \\
\text { Social }(N=34)\end{array}$ & $30(88.2 \%)$ & $28(82.4 \%)$ & $25(73.5 \%)$ & $\begin{array}{c}20 \\
(58.8 \%) \\
\end{array}$ & \\
\hline $\begin{array}{l}\text { Pedagogía } \\
(\mathrm{N}=22)\end{array}$ & $20(86.4 \%)$ & $20(90.9 \%)$ & $17(77.3 \%)$ & $\begin{array}{c}15 \\
(68.2 \%)\end{array}$ & \\
\hline $\begin{array}{l}\text { Universidades } \\
\text { públicas } \\
\text { (todos los } \\
\text { grados) } \mathrm{N}=85\end{array}$ & $75(88.2 \%)$ & $69(81.2 \%)$ & $62(72.9 \%)$ & $\begin{array}{c}53 \\
(62.4 \%)\end{array}$ & \multirow{2}{*}{$\begin{array}{l}\text { públicas vs } \\
\text { privadas } \chi^{2} \\
=15.50_{(4)}, p= \\
.004\end{array}$} \\
\hline $\begin{array}{l}\text { Universidades } \\
\text { privadas } \\
\text { (todos los } \\
\text { grados) } \mathrm{N}=31\end{array}$ & $20(64.5 \%)$ & $24(77.4 \%)$ & $16(51.6 \%)$ & $\begin{array}{c}14 \\
(45.2 \%)\end{array}$ & \\
\hline
\end{tabular}

Fuente: Elaboración propia

En este sentido, el grupo de discusión pone en evidencia el poder de influencia que tiene el profesorado en el momento de decidir el tipo de TFG a realizar, ya que en muchas ocasiones "los TFG están pensados al margen de las necesidades, intereses e inquietudes de los alumnos" (GES1), "siendo los intereses de los equipos de investigación quienes realizan las propuestas temáticas" (GP3). Esta situación, que pudiera parecer perjudicial para el alumnado, es considerada positiva cuando la especialización del profesorado, los proyectos en los que participa, se ponen a disposición del alumnado porque los "TFG de investigación e intervención... [permiten] incorporarles [al alumnado] a nuestros grupos de investigación, en proyectos de investigación que ya tenemos o en proyectos de innovación donde pueden hacer una intervención" (GP3). Se trata de una situación que debe contemplar la "negociación" (GP2) o "pactos de colaboración” (GES2) entre los agentes implicados, docente y estudiante, como parte del proceso de definición de la propuesta. 
Esta valoración es matizada por otra persona integrante del grupo al considerar que la tipología de investigación, habitualmente incluida en las guías docentes, tiene sentido en los grados objeto de estudio si se trata de investigación aplicada, contextualizada, si puede generar un retorno en el contexto laboral. No siempre los proyectos y estudios académicos en los que está inmerso el profesorado podrían servir a esta finalidad, "realmente la modalidad de investigación tendría que estar orientada a la investigación vinculada al entorno laboral, a los contextos de educación formal e informal" (GES3).

En esta línea argumental, se apunta el interés de trabajar en proximidad con el Colegio Profesional porque "el TFG es un trabajo profesional, no un trabajo para publicar artículos ni para proyectar al interior de un grupo de investigación, [ya que] el grado forma profesionales" (GES1). De hecho, se expresa la existencia de "experiencias dramáticas" (GP2) en la realización de TFG en tipologías de artículo científico dada la dificultad de su elaboración por parte del estudiantado por la falta de formación previa en este tipo de trabajos a lo largo del grado.

Una posible solución a esta situación ambivalente podría ser partir de propuestas, ya sean de investigación o revisión teórica, que "se hagan desde el contexto, habiendo identificado las necesidades y, por lo tanto, desde la práctica y desde la profesión, desde el ejercicio" (GES3).

\section{Metodología: la labor tutorial}

Otra de las temáticas que se abordó en el grupo de discusión fue la labor tutorial del profesorado sobre los estudiantes que realizan su TFG. A este respecto, varios participantes coincidieron en asumir que cualquier docente "se supone que está suficientemente cualificado para desarrollarlo” (GP3). Sin embargo, en algunas situaciones "el profesorado asociado puede no tener conocimientos básicos para tutorizar TFG” (GES3). Esta circunstancia se torna problemática cuando el profesorado con dedicación permanente en la universidad tiende a evitar la elección de tutorización de TFG dado el desequilibrio entre las horas de dedicación y la carga docente que se le atribuye. "Se impone el principio de realidad" (GES3), ya que hay condicionantes organizativos y de capacidad docente de los departamentos que son los que determinan las posibilidades de asignación de tutores en función de intereses, capacidades, afinidades, etc. De esta manera a veces "se pervierte" (GM1) lo que significa realmente tutorizar estudiantes de TFG.

La complejidad en la tutorización estriba también en el hecho de ser una tarea individual, con pocos espacios colaborativos para compartir dudas y experiencias, también entre el profesorado. Razón por la cual, no siendo necesaria una formación previa del profesorado, sí se "echa de menos una 
formación en formato de reflexión, compartir experiencias" (GP3). Esta podría estar centrada en "cómo se gestiona el compromiso del estudiante de acuerdo a su TFG" (GES1), refiriéndose a los tiempos de entrega, los tiempos de revisión, "que el profesorado piense sobre cómo es un proceso de TFG" (GES1). El principal problema que surge a este respecto es que cuando se proponen espacios formativos, el profesorado "que más lo necesita no se forma" (GES2).

En otro orden de cosas, uno de los factores que facilita el proceso de tutorización es la continuidad y estabilidad en el profesorado que tutoriza los TFG, lo que contrasta con las situaciones más habituales en las que "te encuentras cada año con un equipo docente nuevo a cargo del TFG con el que hay que empezar de cero" (GP2). El papel de expertos pone en evidencia la necesidad de reclamar "regularidad" (GP1, GP2, GM1) y estabilidad, en el profesorado.

\section{Competencias}

Universidades públicas y privadas incorporan con mayor frecuencia las competencias generales y específicas en comparación con las transversales (Tabla 5), si bien existe una amplia dispersión entre titulaciones. Las competencias generales en el grado de Pedagogía aparecen con mayor frecuencia, en el $63.6 \%$ de las guías, mientras en los grados de Maestro/a tan solo en el $45 \%$.

Por su parte, la presencia en las guías de TFG de las competencias transversales juega un papel secundario. De hecho, solo en los grados de Educación Social encontramos cierta relevancia (35.3\%).

Las universidades públicas tienden a incluir en las guías docentes un amplio abanico de tipos de competencia; las competencias más frecuentes son las específicas $(57.6 \%)$, las generales $(49.4 \%)$ y las básicas $(44.7 \%)$. Las universidades privadas concentran su atención en las competencias específicas $(67.7 \%)$ y generales $(61.3 \%)$.

El grupo de discusión insiste en la importancia del TFG como instrumento que testa la totalidad de las competencias que se pretenden alcanzar en el grado. Así, es considerado un proyecto de envergadura que debe responder a las competencias del grado. De hecho:

El objetivo fundamental o una de las competencias fundamentales que se debe trabajar en el TFG es que los alumnos demuestren todas aquellas competencias instrumentales, generales, específicas que han ido adquiriendo a lo largo de los cuatro años del grado (GP1). 
Tabla 5

Tratamiento de las competencias en las guías de TFG en los grados del área educativa de las universidades españolas

\begin{tabular}{llllll}
\hline & Básicas & Generales & $\begin{array}{c}\text { Transversales/ } \\
\text { Claves }\end{array}$ & Específicas & $\begin{array}{c}\text { Sin } \\
\text { clasificar }\end{array}$ \\
\hline $\begin{array}{l}\text { Maestro/a } \\
(\mathrm{N}=60)\end{array}$ & $29(48.3 \%)$ & $27(45 \%)$ & $10(16.7 \%)$ & $38(63.3 \%)$ & $2(3.3 \%)$ \\
\hline $\begin{array}{l}\text { Educación } \\
\text { Social (N=34) }\end{array}$ & $11(32.4 \%)$ & $20(58.8 \%)$ & $12(35.3 \%)$ & $18(53 \%)$ & $2(5.9 \%)$ \\
\hline $\begin{array}{l}\text { Pedagogía } \\
(\mathrm{N}=22)\end{array}$ & $7(31.8 \%)$ & $14(63.6 \%)$ & $4(18.2 \%)$ & $14(63.6 \%)$ & $5(22.7 \%)$ \\
\hline $\begin{array}{l}\text { Universidades } \\
\text { públicas } \\
(\mathrm{N}=85)\end{array}$ & $38(44.7 \%)$ & $42(49.4 \%)$ & $20(23.5 \%)$ & $49(57.6 \%)$ & $6(7.1 \%)$ \\
\hline $\begin{array}{l}\text { Universidades } \\
\text { privadas } \\
(\mathrm{N}=31)\end{array}$ & $9(29 \%)$ & $19(61.3 \%)$ & $4(12.9 \%)$ & $21(67.7 \%)$ & $3(9.7 \%)$ \\
\hline
\end{tabular}

Fuente: Elaboración propia

\section{Relación entre el TFG y el prácticum}

La mayor parte de guías docentes, en universidades públicas y privadas, no indican relación entre prácticum y TFG en los grados de Educación Social y Maestro/a (60.7\% y 74.4\%, respectivamente, de las universidades públicas y $76.2 \%$ y $100 \%$, respectivamente, en las universidades privadas). En el grado de Pedagogía, la frecuencia en la que el prácticum se considera como espacio de intervención y/o de análisis desde el TFG se sitúa en un $61.1 \%$ en las universidades públicas y en un $50 \%$ en las privadas. La consideración de que sea la misma persona quien tutoriza al estudiantado en el prácticum y el TFG es escasa en las universidades públicas, y nula en las privadas.

El grupo de discusión pone de manifiesto algunos inconvenientes en la relación del prácticum y el TFG. Así, algunos expertos consideran que los productos de ambos espacios formativos podrían sufrir solapamientos, viéndose minusvalorada la tipología de TFG de intervención ya que "mientras el estudiante está realizando el TFG, al mismo tiempo está realizando el prácticum II, entonces intenta... que lo que hace en el TFG no sea en el mismo contexto en el cual realiza su prácticum" (GP2).

En esta relación entre ambos planos formativos, el TFG parece salir perdiendo porque gana protagonismo el contexto donde se realiza la intervención (el prácticum): 
El estudiante está planificando una intervención, aplicándola, desarrollándola y evaluándola, y muchas veces..., el producto del TFG, finalmente lo que se presenta, tiene menor implicación en el contexto que la propia intervención que se ha hecho en el prácticum (GP2).

En esa misma línea se esgrimen argumentos al observar dificultades en la identificación del perfil profesional y la transferencia de conocimientos académicos a la práctica cuando el TFG se propone en el mismo contexto que el prácticum porque:

Hay alumnado con serias dificultades para saber qué hace el perfil profesional en la práctica, porque lo tienen muy distorsionado, cuando van a las prácticas tienen dificultad para transferir lo que han aprendido en el grado a su experiencia práctica (GES1).

Quizás, "si la persona que tutoriza el prácticum y el TFG fuera la misma, estos riesgos disminuirían” (GES3).

\section{EVALUACIÓN}

\section{Agentes evaluadores}

Los principales agentes encargados de evaluar el TFG son el tutor/a y los tribunales de evaluación (Tabla 6). En las universidades públicas es más frecuente que el tutor/a sea uno de los agentes de evaluación (89.4\% de las titulaciones), mientras que en las universidades privadas los tribunales obligatorios son notablemente más frecuentes (están presentes en el 80.6\% de las titulaciones). Estas diferencias entre las universidades públicas y las privadas son estadísticamente significativas $\left(\chi^{2}=18.85_{(6)}, p=.004\right)$. Sin embargo, no hay diferencias significativas entre las titulaciones $\left(\chi^{2}=11.80_{(12)}\right.$, $p=.462)$. 


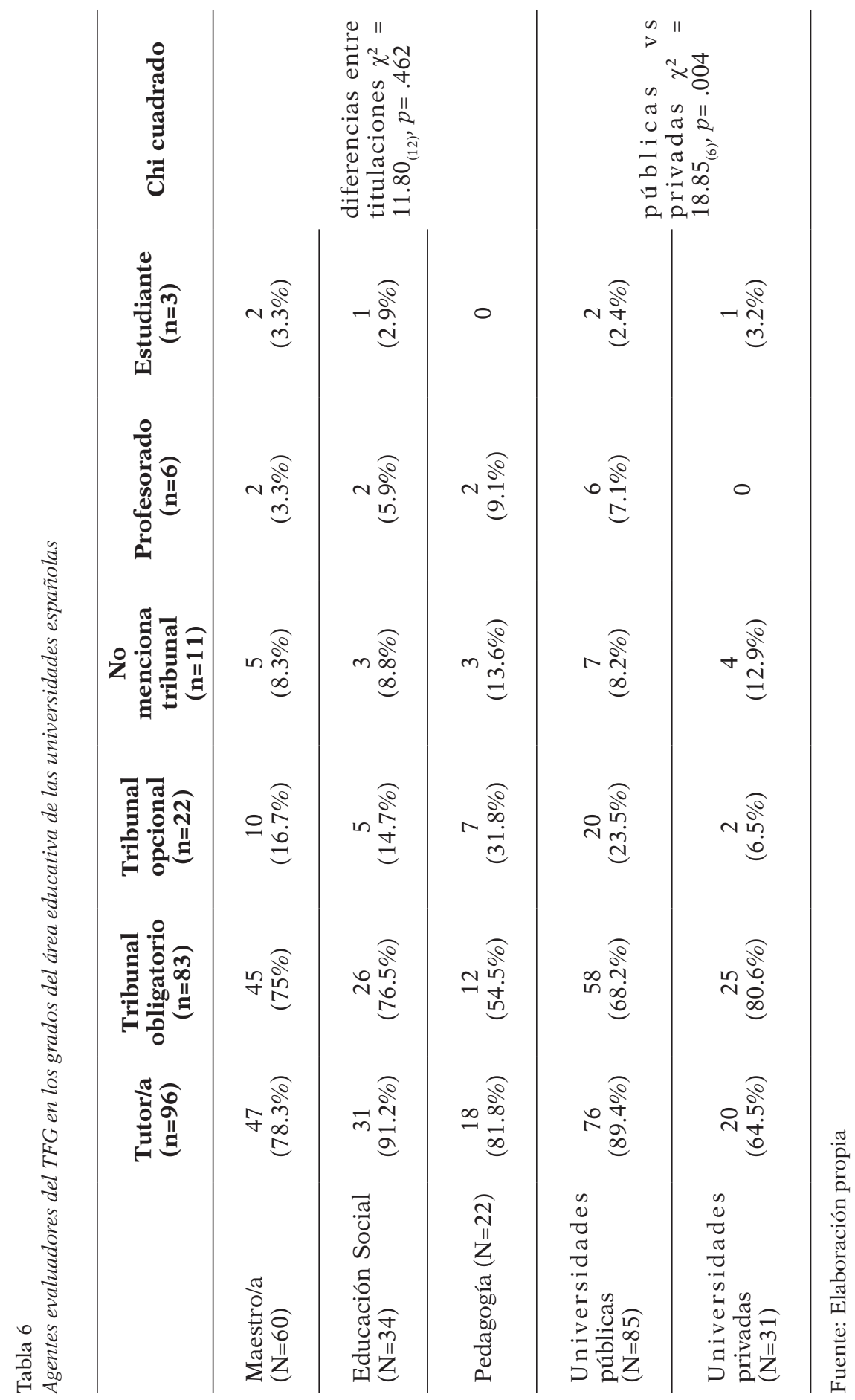




\section{ELEMENTOS EVALUADOS}

Los principales elementos que se valoran en la calificación de los TFG (Tabla 7), son el informe final y la defensa ante el tutor o tutora, o ante tribunal. Los informes intermedios del TFG se evalúan en un número menor de ocasiones (el $50.6 \%$ de los grados en las universidades públicas y el 71.0\% en las universidades privadas). Además, los informes iniciales del TFG apenas tienen influencia en la calificación. Las diferencias entre las universidades públicas y las privadas en los elementos evaluados en los TFG son estadísticamente significativas $\left(\chi^{2}=17.56_{(5)}\right.$, $p=.004)$, mientras que las diferencias en este aspecto entre las titulaciones no lo son $\left(\chi^{2}=8.42_{(10)}, p=.588\right)$.

Tabla 7

Elementos evaluados del TFG en los grados del área educativa de las universidades españolas

\begin{tabular}{|c|c|c|c|c|c|}
\hline & $\begin{array}{c}\text { Informe } \\
\text { final } \\
(n=111)\end{array}$ & $\begin{array}{c}\text { Defensa } \\
(n=105)\end{array}$ & $\begin{array}{l}\text { Informe } \\
\text { de } \\
\text { proceso } \\
(n=65)\end{array}$ & $\begin{array}{l}\text { Informe } \\
\text { inicial } \\
(n=13)\end{array}$ & Chi cuadrado \\
\hline $\begin{array}{l}\text { Maestro/a } \\
(\mathrm{N}=60)\end{array}$ & $\begin{array}{c}58 \\
(96.7 \%)\end{array}$ & $54(90 \%)$ & $30(50 \%)$ & $6(10 \%)$ & \multirow{3}{*}{$\begin{array}{l}\text { Diferencias entre } \\
\text { titulaciones } \chi^{2}= \\
8.42_{(10)}, p=.588\end{array}$} \\
\hline $\begin{array}{l}\text { Educación } \\
\text { Social }(N=34)\end{array}$ & $\begin{array}{c}33 \\
(97.1 \%)\end{array}$ & $32(94.1 \%)$ & $19(55.9 \%)$ & $4(11.8 \%)$ & \\
\hline $\begin{array}{l}\text { Pedagogía } \\
(\mathrm{N}=22)\end{array}$ & $\begin{array}{c}20 \\
(90.9 \%)\end{array}$ & $19(86.4 \%)$ & $16(72.7 \%)$ & $3(13.6 \%)$ & \\
\hline $\begin{array}{l}\text { Universidades } \\
\text { públicas (todos } \\
\text { los grados) } \\
(\mathrm{N}=85)\end{array}$ & $\begin{array}{c}84 \\
(98.8 \%)\end{array}$ & $78(91.8 \%)$ & $43(50.6 \%)$ & $\begin{array}{c}11 \\
(12.9 \%)\end{array}$ & \multirow{2}{*}{$\begin{array}{l}\text { públicas vs } \\
\text { privadas } \chi^{2}= \\
17.56_{(5)}, p=.004\end{array}$} \\
\hline $\begin{array}{l}\text { Universidades } \\
\text { privadas (todos } \\
\text { los grados) } \\
(\mathrm{N}=31)\end{array}$ & $\begin{array}{c}27 \\
(87.1 \%)\end{array}$ & $27(87.1 \%)$ & $22(71 \%)$ & $2(6.5 \%)$ & \\
\hline
\end{tabular}

Fuente: Elaboración propia

El grupo de discusión pone sobre la mesa la necesidad de considerar los tribunales de evaluación como obligatorios (GES1, GES2, GES3, GP3) ya que desarrollan al estudiantado profesionalmente:

Tiene que utilizar competencias que después son necesarias en la vida profesional. El enfrentamiento a una situación de evaluación... de manera individual, y ante un tribunal que no se ha visto nunca, creo que es necesario, que es adecuado (GES3). 
De hecho, parece importante que "antes de salir al mundo profesional se enfrenten a un tribunal, con preguntas, con una situación de estrés... creo que es un aprendizaje importante el poder defender algo que tú has hecho delante de un tribunal” (GES2). La defensa del TFG ante tribunal se convierte en una experiencia de aprendizaje pues "aprendes también a enfrentarte a una situación que es defender el saber y responder a las preguntas que el tribunal te hace en ese momento" (GP3), es una oportunidad para potenciar "la capacidad de construir colectivamente la identidad profesional de nuestros alumnos" (GES1); de ahí su importancia en el proceso formativo.

El principal inconveniente para hacer efectivo este procedimiento de evaluación es la "dificultad que supone organizar los tribunales de TFG, la carga de trabajo que puede suponer para el profesorado, e incluso las dificultades que tienen que ver con relaciones conflictivas entre profesorado, desavenencias" (GP3) entre los miembros del tribunal que pudieran afectar las valoraciones y calificación del alumnado tutorizado. Para favorecer que los tribunales sean lo más equitativos posible, se propone que tengan representación de distintas áreas de conocimiento y diversidad de enfoques epistemológicos, además del uso de rúbricas para la evaluación.

Sin embargo, y a pesar de las virtudes identificadas en la defensa de los TFG ante tribunal, se viene observando que el alumnado "prefiere quedarse con su 7 antes que exponerse a un tribunal" (GES3), hecho que anima al grupo de discusión a confirmar la necesidad de su obligatoriedad.

\section{DISCUSIóN}

Tras el análisis de contenido de las guías docentes y la celebración del grupo de discusión, una de las primeras conclusiones que podemos extraer es la preocupante similitud en el enfoque que las universidades adoptan sobre los TFGs de los grados de educación, una similitud especialmente notable en el caso de los dos Grados de Maestro/a en Educación Infantil y Educación Primaria, pese a que se trata de perfiles profesionales con identidades y ámbitos de intervención claramente diferenciados. Sin embargo, sí existen diferencias significativas en numerosos aspectos de la implementación del TFG entre universidades públicas y privadas. Las universidades públicas otorgan al TFG un número de créditos significativamente inferior a las universidades privadas. A este respecto, el grupo de discusión valora que debe llevarse a cabo una reconsideración del número de créditos que se otorga al TFG, para que sea más acorde al nivel de exigencia curricular planteado. En la misma línea se expresan Anderson et al. (2008) cuando aluden a la escasa disposición de tiempo para su ejecución.

Asimismo, encontramos diferencias relativas a la diversidad de tipologías de TFG entre universidades públicas y privadas, de manera que las universidades públicas contemplan habitualmente una variedad mayor de tipos de TFG 
que las privadas. A este respecto, el grupo de discusión valora positivamente disponer de un abanico amplio, insistiendo en la relevancia de responder al perfil profesional, si bien se genera cierta controversia sobre la idoneidad de la tipología investigadora en determinados grados (Grados de Magisterio y Educación Social), dado que sus planes de estudios no incorporan competencias investigadoras. Este planteamiento concuerda con el de Cuthbert (1995), al reconocer que se trata del primer momento en el que se ponen a prueba estas competencias, lo que considera una oportunidad para que el estudiantado incorpore nuevas habilidades investigadoras. Desde el grupo de discusión se observa un claro interés por aproximar el TFG al contexto laboral. Sin embargo, no parece que establecer una relación entre el prácticum y el TFG, es decir, una tutorización del mismo profesor/a o una intervención/investigación relacionada con el centro de prácticas, sea la solución. Relación de escasa frecuencia en guías docentes y ampliamente cuestionada por el grupo de discusión. Sin embargo, como equipo investigador valoramos la necesidad de explorar en mayor profundidad este tema, escasamente analizado y quizás propicio si la definición de las guías docentes del prácticum y del TFG ponen el acento en evitar solapamientos.

En atención a la evaluación, contrasta la relevancia que le otorgan las universidades públicas a incorporar al tutor como agente, con su menor frecuencia en las universidades privadas, que optan por una presencia significativamente mayor de tribunales. Complementariamente, la autoevaluación es residual o inexistente en las guías docentes de universidades públicas y privadas, a pesar de ser considerada relevante como espacio de reflexión del estudiante sobre su propio trabajo (Fraile et al., 2018). En esta línea, Tejada y Ruiz (2016) aluden a la necesidad de incorporar, eso sí, de forma ponderada, las perspectivas de diferentes agentes en la evaluación.

Otros hallazgos relevantes constatan que las guías docentes del TFG concentran su atención principalmente en la consecución de las competencias generales y específicas, siendo menos frecuente su alusión a las transversales. En este sentido, el grupo de discusión considera que el TFG debe ser un instrumento que evidencie si las competencias del grado han sido adquiridas. Demandas globales, que no son fáciles de atender (Rosenmund, 2006). De hecho, esta amplitud de miras, constatada en gran número de guías docentes, puede quedar como una declaración de intenciones, dada la dificultad para incorporar en la evaluación la totalidad de las competencias del grado, indicio de la dificultad de trasladar a la práctica estos determinantes curriculares.

Sorprende reparar en cómo la labor tutorial no queda reflejada en las guías. El grupo de discusión reflexiona sobre la falta de cualificación de determinados colectivos de profesorado, especialmente el menos experimentado o aquel con escasa formación investigadora. También se insiste en la necesidad de revisar la profunda individualización del profesorado, falto de espacios de reflexión colectiva, así como en la necesidad de conectar, de forma contextualizada, con los intereses y realidad del estudiante (Ortega y Pagès, 2018). En línea con Molina 
et al. (2020), el profesorado debería redefinir su rol. Complementariamente, se alude a su escasa estabilidad en la impartición de esta asignatura, lo que dificulta la generación de criterios compartidos y el avance en procesos reflexivos sobre la acción tutorial.

Así, podemos concluir que el diseño curricular de la asignatura de TFG debe tornarse colectivo, someterse a estudio y reflexión con objeto de dotar a la asignatura de un contenido curricular compartido y actualizado. Complementariamente, se constata la consideración generalizada del TFG como un proceso de generación de conocimiento (Díaz-Vázquez et al., 2018), un proyecto vital y profesional (Brew, 2007), que sin embargo contrasta con la escasez de créditos asignados, con los problemas de cualificación en una parte del profesorado o con determinantes curriculares en las guías docentes difícilmente trasladables a la práctica.

Si una potencialidad de esta investigación es haber accedido al universo de las guías docentes de la asignatura de TFG en los grados de Magisterio, Educación Social y Pedagogía de las universidades españolas, podríamos considerar una limitación no haber incluido en el análisis otros documentos complementarios: reglamentos del TFG, programas docentes, guías de elaboración, informes, anexos, rúbricas.

Finalmente, una futura línea de investigación podría conducirnos al análisis del currículum en acción, es decir, al estudio de la práctica curricular docente, un estudio que requeriría incorporar al profesorado y estudiantado como sujetos de análisis. La tensión curricular que se genera entre el currículum prescrito y el currículum que se traslada a la práctica, sin duda podría permitirnos profundizar sobre el sentido de esta asignatura y valorar si algunas de las evidencias, también indicios, puestos de relieve en este artículo, se sostienen en la práctica. 


\section{REFERENCIAS BIBLIOGRÁFICAS}

Anderson, C., Day, K., \& McLaughlin, P. (2008). Student perspectives on the dissertation process in a masters degree concerned with professional practice. Studies in Continuing Education, 30(1), 33-49. https://doi. org/10.1080/01580370701841531

Bauman, Z., \& Leonci, T. (2018). Generación líquida: Transformaciones en la era 3.0. Paidós.

Brew, A. (2007). Aproaches to the scholarships of teaching and learning. En A. Brew \& J. Sach, Transforming a University. The scholarships of teaching and learning in practice. University Press.

Carlós, L., \& Telmo, D. (2000). El análisis de contenido: su presencia y uso en las ciencias sociales. https://kutt.it/BdZyS4

CRUE (2020). Listado de Universidades Españolas. https://www.crue.org/ universidades/

Cuthbert, K. (1995). Project planning and the promotion of self regulated learning: From theory to practice. Studies in Higher Education, 20(3), 267-277. https:// doi.org/10.1080/03075079512331381545

Díaz-Vázquez, R., García-Díaz, A, Maside, J.M., \& Vázquez-Rozas, E. (2018). El Trabajo Fin de Grado: fines, modalidades y estilos de tutorización. REDU. Revista de Docencia Universitaria, 16(2), 159-175. https://doi.org/10.4995/redu.2018.10178

Díez, L. (2015). Las responsabilidades del profesor en la dirección de los trabajos de fin de grado. Docencia y Derecho. Revista para la Docencia Jurídica Universitaria, 9, 1-19.

Fraile, J., Pardo, A., \& Panadero, E. (2018). Autoevaluación y autocalificación en el Grado en Ciencias de la Actividad Física y del Deporte: Estudio censal de las guías docentes. Profesorado. Revista de Currículum y Formación, 22(3), 163-182. https://doi.org/10.30827/profesorado. v22i3.7997
Fretwell, D. (2003). AA framework for evaluating vocational education and training (VET). European Journal of Education, 38(2), 177-190 https://doi. org/10.1111/1467-3435.00137

Gervilla-Castillo, E. (2004). Buscando valores: El análisis de contenido axiológico. Perfiles educativos, 26(103), 95-110.

Healey, M., Lannin, L., Stibbe, A., \& Derounian, J. (2013). Developing and enhancing undergraduate final-year projects and dissertations. The Higher Education Academy.

Jawitz, J., Moore, R., \& Shay, S. (2002). Management and assessment of final year projects in engineering. International Journal of Engineering Education, 18(4), 472-478.

Krippendorff, K. (1990). Metodología del análisis de contenido. Teoría y Práctica. Paidós.

Manzanares-Moya, A., \& SánchezSantamaría, J. (2016). Aplicaciones de la tutoría y la evaluación para activar competencias en los Trabajos Fin de Grado y Máster. En A. Mateo Jiménez y A. Manzanares-Moya (Dirs.), Mejores maestros, mejores educadores: innovación y propuestas en Educación (pp. 195-216). Aljibe.

Mateo, J., Escofet, A., Martínez-Olmo, F., Ventura, J., \& Vlachopoulos, D. (2012). Evaluation Tools in the European Higher Education Area (EHEA): an assessment for evaluating the competences of the Final Year Project in the social sciences. European Journal of Education, 47(3), 435-447. http://doi.org/10.1111/j.14653435.2012.01536.x

Molina, M.D., Rodríguez, J., \& Colmenero, M.J. (2020). Importancia de la tutorización para el éxito del Trabajo Fin de Grado. Revista Complutense De 
Educación, 31(2), 241-250. https://doi. org/10.5209/rced.63120

Ortega, D., \& Pagès, J. (2018). Género y formación del profesorado: análisis de las Guías Docentes del área de Didáctica de las Ciencias Sociales. Contextos Educativos. Revista de Educación, 21, 53-66. https://doi.org/10.18172/con.3315

Palomares-Montero, D., \& ChisvertTarazona, M.J. (2014). Ética y empresa en el espacio universitario: el emprendimiento social en las universidades públicas como vehículo facilitador de la equidad social. REDU: Revista de Docencia Universitaria, 12(2), 205-230.

Perelló, S. (2009). Metodología de la Investigación Social. Dykinson.

Real Decreto 1393/2007, de 29 de octubre, por el que se establece la ordenación de las enseñanzas universitarias oficiales. Boletín Oficial del Estado, núm. 260, de 30 de octubre de 2007.

Rekalde, I. (2011). ¿Cómo afrontar el Trabajo fin de grado? Un problema o una oportunidad para culminar con el desarrollo de las competencias. Revista Complutense de Educación, 22(2), 179-193. https://doi.org/10.5209/rev_ RCED.2011.v22.n2.38488

Rodríguez, J., Molina, M. D., \& Colmenero, M. J. (2019). La voz de los estudiantes de la titulación de Educación Primaria sobre el Trabajo Fin de Grado. Educação e Pesquisa, 45(14), 1-21. https://doi. org/10.1590/s1678-4634201945200829

Rosenmund, M. (2006). The current discourse on curriculum change: a comparative analysis of National Reports on Education. En A. Benavot \& C. Braslavsky (Eds.), School Knowledge in Comparative and Historical Perspective. Changing Curricula in Primary and Secondary Education. Springer.

Sánchez, A., Olmos, P., Torrado, M., \& García, J. (2016). Trabajos fin de grado $y$ de postgrado. Guía práctica para su elaboración. Aljibe.

Sánchez-Santamaría, J. (2017). Evaluar y valorar el TFG: la evaluación como aprendizaje. Universidad de Castilla-La Mancha.

Tejada, J., \& Ruiz, C. (2016). Evaluación de competencias profesionales en Educación Superior: Retos e implicaciones. Educación XX1, 19(1), 17-38. https://doi.org/10.5944/ educXX1.12175

Vera, J., \& Briones, E. (2015). Students' perspectives on the processes of supervision and assessment of undergraduate dissertations. Cultura $y$ Educación, 27(4), 726-765. https://doi.or $\mathrm{g} / 10.1080 / 11356405.2015 .1089391$ 


\section{Anexo 1} discusión

Unidades de contexto y guion de las preguntas planteadas en el grupo de

Características generales

Tipología y formato

Competencias

Metodología: acción tutorial

Relación con el prácticum

Evaluación
“¿Qué implicaciones tiene para el alumnado y el proceso de tutorización, que se otorguen 6 créditos al TFG?, circunstancia que se produce con más claridad en Magisterio y Educación Social, y en menor medida en Pedagogía, y en universidades públicas, antes que en privadas"

"La revisión ha mostrado que las tipologías más frecuentes para la realización de un TFG individual son: investigación, revisión teórica, intervención y proyecto técnico, ¿qué tipología sería más adecuada para demostrar la adquisición de las competencias que se pretenden en un TFG de un grado de educación?"

"En la revisión realizada no encontramos referencias relativas a tutorización, asignación docente, carga docente, formación previa, continuidad/estabilidad ¿deseáis realizar alguna aportación?"

"Solo un cuarto de las universidades relaciona el TFG a la realización del prácticum en los grados de Magisterio y Educación Social, y alrededor de la mitad en el caso de Pedagogía, ¿qué tipo de relación consideráis más propicia?”

“¿Cuál debería ser el papel del tutor o tutora en la evaluación? ¿El tribunal en los TFG de educación debería ser obligatorio, optativo o incluso podría ser eliminado?"

Fuente: Elaboración propia 


\section{PERFIL ACADÉMICO Y PROFESIONAL DE LOS AUTORES}

María José Chisvert-Tarazona. ORCID: https://orcid.org/0000-0002-5533-8100

Profesora contratada doctora del Departamento de Didáctica y Organización Escolar de la Universitat de València. Entre sus líneas de investigación destaca la formación profesional, la formación en el trabajo y la formación superior. E-mail: Maria.Jose.Chisvert@uv.es

Raúl Tárraga-Mínguez. ORCID: https://orcid.org/0000-0002-4458-5763

Profesor titular de universidad del Departamento de Didáctica y Organización Escolar de la Universitat de València. Sus líneas de investigación se centran en la formación del profesorado y la educación inclusiva. E-mail: Raul.Tarraga@uv.es

Alicia Ros-Garrido. ORCID: https://orcid.org/0000-0002-6968-0900

Profesora contratada doctora del Departamento de Didáctica y Organización Escolar de la Universitat de València. Entre sus líneas de investigación destaca la formación del profesorado, la formación profesional y la formación en el trabajo. E-mail: Alicia.Ros@uv.es

Davinia Palomares-Montero. ORCID: https://orcid.org/0000-0002-3671-2195

Titular de Universidad del Departamento de Didáctica y Organización Escolar de la Universitat de València. Entre sus líneas de investigación destaca la formación profesional, la formación en el trabajo y el emprendimiento social en el contexto universitario. E-mail: Davinia. Palomares@uv.es

Fecha Recepción del Artículo: 14. Octubre. 2020

Fecha Modificación del Artículo: 30. Enero. 2021

Fecha Aceptación del Artículo: 31. Enero. 2021

Fecha Revisión para Publicación: 23. Febrero. 2021 